\title{
Prolonged Obstructed Labor Is an Uncommon Presentation of a Giant Bladder Calculus: A Case Report and Literature Review
}

\author{
B. M. Abubakar1 ${ }^{*}$, A. Abdulkadir ${ }^{2}$, A. A. Atterwahmie' ${ }^{3}$, A. A. Panti ${ }^{4}$, A. I. Maina ${ }^{5}$ \\ ${ }^{1}$ Department of Surgery, Federal Medical Centre, Nguru, Nigeria \\ ${ }^{2}$ Department of Surgery, Bayero University/Aminu Kano Teaching Hospital, Kano, Nigeria \\ ${ }^{3}$ Department of Obstetrics and Gynaecology, Federal Medical Centre, Nguru, Nigeria \\ ${ }^{4}$ Department of Obstetrics and Gynaecology, Usmanu Danfodiyo University Teaching Hospital, Sokoto, Nigeria \\ ${ }^{5}$ Birniwa General Hospital, Birniwa, Nigeria \\ Email: ^bgmustapha2003@yahoo.co.uk, ^bgmustapha@unimaid.edu.ng
}

How to cite this paper: Abubakar, B.M., Abdulkadir, A., Atterwahmie, A.A., Panti, A.A. and Maina, A.I. (2019) Prolonged Obstructed Labor Is an Uncommon Presentation of a Giant Bladder Calculus: A Case Report and Literature Review. Open Journal of Urology, 9, 77-83.

https://doi.org/10.4236/oju.2019.94009

Received: January 27, 2019

Accepted: April 23, 2019

Published: April 26, 2019

Copyright $\odot 2019$ by author(s) and Scientific Research Publishing Inc. This work is licensed under the Creative Commons Attribution International License (CC BY 4.0).

http://creativecommons.org/licenses/by/4.0/

cC) (7) Open Access

\begin{abstract}
Prolonged obstructed labor is a critical intrapartum emergency. This intricacy is, however, unusual as a primary presentation of a giant bladder calculus. We report a case of a 25-year-old Para $6+1$, who presented with a history of labor pains of 72 hours duration. She had background lower urinary tract symptoms with supra-pubic swelling and hematuria of 2 years duration. She had a single antenatal visit at 32 weeks however; no obstetric ultrasound scan was done before she went into labor. Examination revealed a highly placed fetal head with increase fetal heart rate. There was a hard mass bulging under the anterior vaginal wall. The diagnosis was a prolonged obstructed labor secondary to bladder mass with fetal distress was made. She had a caesarean section (CS) with the delivery of fresh stillborn. The bi-manually palpable bladder mass persisted post-CS. Her abdominopelvic ultrasound scan and abdominal X-ray post-CS revealed giant vesical calculus with obstructive uropathy. Urine microscopy culture and sensitivity yielded $E$. coli sensitive to Ciprofloxacin; other laboratory investigations were normal. She was treated for UTI and 2 weeks post-CS, she had opened cystolithotomy. The stone weighed $536 \mathrm{~g}$. Her postoperative recovery was uneventful.
\end{abstract}

\section{Keywords}

Prolonged Obstructed Labor, Giant Calculus, Primary Presentation

\section{Introduction}

Prolonged obstructed labor is a critical intrapartum emergency essentially seen 
in impoverished populations that have deficiencies in the modern healthcare delivery [1]. Short of appropriate immediate intervention, the outcome includes stillbirth, maternal morbidity and mortality. Urolithiasis is among the major sources of urological emergencies in pregnancy. The finding of vesical calculus in pregnancy during routine obstetric ultrasound scanning was documented in the literature. These are most often small-sized and asymptomatic or linked with recurrent UTI [2]. They are usually managed conservatively until delivery. An appraisal on prolonged obstructed labor as a primary presentation of a giant bladder calculus is scarce in the literature. Notwithstanding, very few cases that are centuries old were reported in the pieces of literature from the developed countries [3] [4]. Some relatively recent sporadic cases from communities deficient in healthcare delivery were reported in the chronicles [1] [5] [6]. Nonetheless, calculus of $536 \mathrm{~g}$ as a cause of prolonged obstructed labor is unheard-of in the medical documentation from our literature search. Our patient was a 25-year-old Para $6+1$ who presented with prolonged obstructed labor due to a giant vesical calculus that necessitated delivery of fresh stillborn by CS. The patient had consented for this report.

\section{Case Report}

The patient was a 25-year-old Para $6+1$, who presented with a history of labor pains for 72 hours at 39 weeks gestation. She had spontaneously ruptured of membranes about 24 hours prior to presentation and had excessive urge to bear down. The labor pain evolved into an unrelenting constant generalized abdominal pain that compelled her presentation. No associated vaginal bleeding, however, she had not been perceiving fetal movements. She had difficulty of passing urine that was characterized predominantly by irritating lower urinary tract symptoms of 10 years duration. There was an associated intermittent terminal painful hematuria with progressive supra-pubic swelling of two years duration. No upper urinary tract symptoms.

She had a single antenatal visit at 32 weeks gestation and was unable to do the requested obstetric ultrasound before labor. All her previous pregnancies were un-booked, and the deliveries were at home, said to be uneventful. Her last confinement was 2 years prior to presentation and was uneventful.

She was examined and found to have a highly placed fetal head with symphysio-fundal height of $42 \mathrm{~cm}$ above pubic symphysis. The fetal heart rate was 182/minute, Vaginal examination revealed a hard mass bulging under the anterior vaginal wall. The cervix was fully dilated and the station was -2 .

The diagnosis of prolonged obstructed labor secondary to bladder mass with fetal distress was made by the medical officer at presentation and thus planned for an emergency CS. She was resuscitated and was eventually delivered by CS to a singleton fresh stillborn fetus. The huge bladder mass was affirmed intra-operatively but was not tempered with at that setting due to lack of expertise and suspicious of bladder tumor. 
The review by urologist one week post-CS revealed a well preserved lady with healed midline suprapubic scar. There was a bi-manually palpable hard, regular and mobile bladder mass that extends $6 \mathrm{~cm}$ above pubic symphysis. Her abdominopelvic ultrasound scan revealed giant vesical calculi with grade 2 to 3 obstructive uropathy. X ray of the region of Kidney, ureter and bladder (KUB) confirmed huge bladder calculus no other stone seen (Figure 1). Urine M/C/S yielded E. coli sensitive to Ciprofloxacin with numerous red blood and pus cells; other laboratory investigations were normal. She was treated for UTI and 2 weeks post-CS had opened cystolithotomy. Intraoperative findings were that of huge bladder stone (Figure 2) weighing $536 \mathrm{~g}$ (Figure 3). The bladder wall was thickened with hyperemic mucosa (Figure 4). There was an adequate bladder neck. The histology of the bladder mucosal biopsies showed features of chronic inflammation with Schistosomal ova. Her post-operative care was uneventful; except for a transient frequency on the removal of the catheter that regressed at follow-up.

\section{Discussion}

The existence of calculus in the urinary tract is an affirmed ubiquitous urological lesion. There is what seems to be changed in the pattern of presentation of urinary stone in general, from lower urinary tract stone to upper urinary tract stone but this change is mainly observed in the developed nations. Urolithiasis has been attested as a major cause of urological emergencies in pregnancy; particularly the ureteric calculus. Vesical calculi, on the contrary, are often asymptomatic

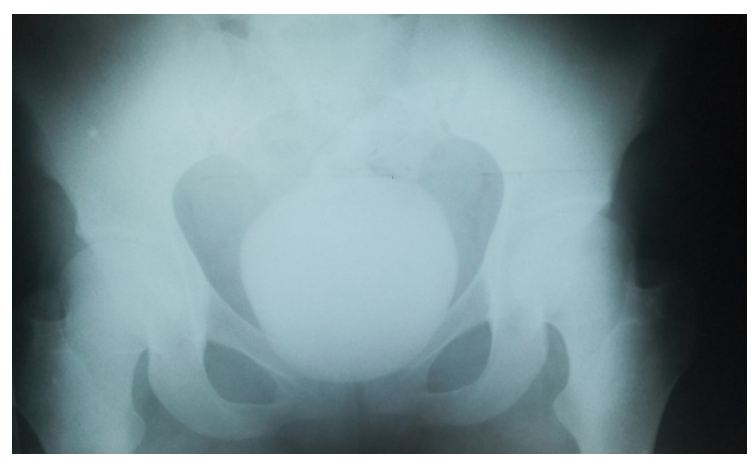

Figure 1. Plain abdominal $\mathrm{X}$ ray (KUB) showing huge bladder calculus no other stone seen.

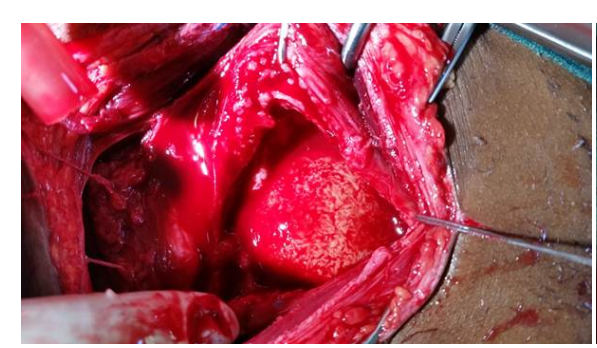

(a)

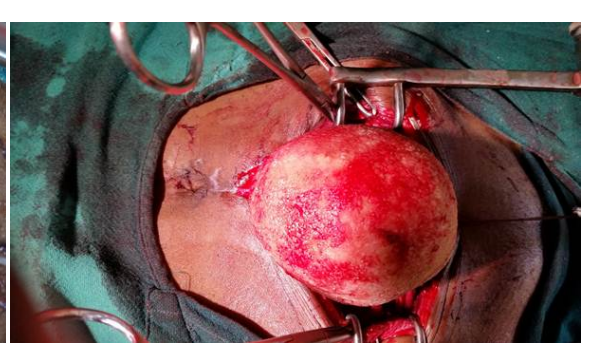

(b)

Figure 2. (a) Intra operative findings of huge bladder stone; (b) Intra operative findings of huge bladder stone. 


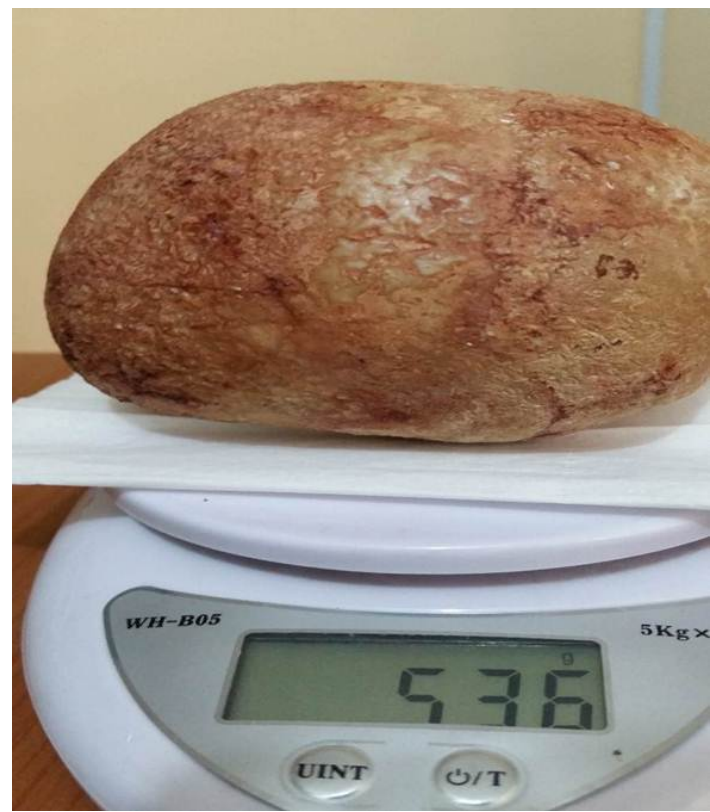

Figure 3. Huge bladder stone weighing $536 \mathrm{~g}$.

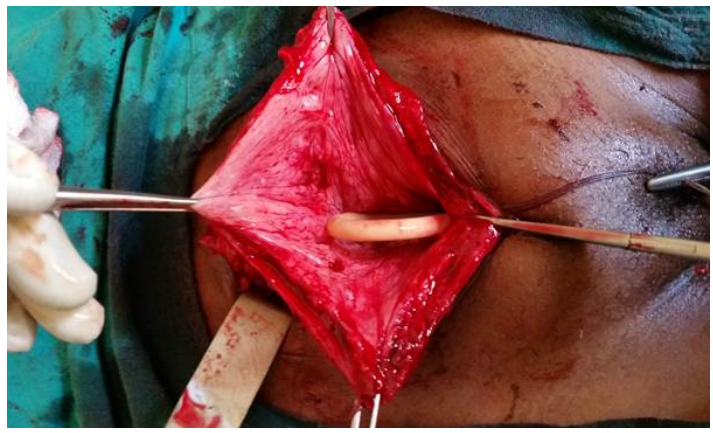

Figure 4. Intra-operative picture showing thickened bladder wall and hyperemic mucosa.

found at routine antenatal obstetric ultrasound scan or following recurrent UTI [2] [7]. The incidence of the vesical calculi in pregnancy was reported to be in the vicinity of 1 in 2000 - 3000 pregnancies [8]. Prolonged obstructed labor as a primary presentation of a vesical calculus is a marker of the sober deficiencies in the healthcare delivery and societal underdevelopment. Consequently, the rare reports were from developing parts of the world notably parts of Indian [1] [6] [9] [10] and some countries in Africa [5] [11] [12]. Notwithstanding the remarkable global advances in healthcare delivery, these settings share a common unabated denominator of public healthcare hurdles [13]. The very few isolated cases from the developed communities date back to the 19th and early 20th centuries [13] [14]. In these developed communities, the incidence of bladder stone has been on the decline since the 19th century [12]. Our search of the literature showed no vesical calculus as a primary presentation of prolonged labor matched the weight of $536 \mathrm{~g}$ in the index patient.

Our patient had 6 un-booked pregnancies with LUTS for 10 years coincided 
with other presentations [1] [11] [15]. These concurred with poor health-seeking behavior linked with ignorance, lack of access or presumed problems of affordability in these settings. Most of the patients that were un-booked presented as an intra-partum emergency. The index case was booked saved no antenatal visit and was at home for 24 hours whilst in labor. The ultrasound scan or cystoscopy required for the diagnoses were however inaccessible in the center at the time of our patient's presentation. In pregnancy, diagnostic imaging which emits ionizing radiations is linked to teratogenic hazard and childhood malignancies. These risks rely on the dose of radiation and gestational age. Ultrasound and Magnetic resonance imaging (MRI) are non-ionizing as such they are the imaging technique of choice in pregnancy. X-ray is used in pregnancy when is eminently essential based on EAU guideline [16]. Minimum (low) dose Abdominal CT protocols are presently promoted for used in pregnancy only as a last choice. This has maximum foetal radiation dose of $49 \mathrm{mGy}$ (mean $=8 \mathrm{mGy}$ ), and for this there is no evidence that a procedure using routine parameters $(<50 \mathrm{mGy})$ at any time of the gestation will result in spontaneous miscarriage or developmental abnormalities [17]. The abdominopelvic ultrasound scan and X-ray were only done in this patient post-CS; MRI and CT scan were unavailable at the hospital.

Other booked patients with an early presentation did well to cystolithotomy with succeeding vagina delivery at term [7]. Trans-vaginal vesicostomy with its pros and cons following vaginal delivery has been described [15]. CS and immediate cystolithotomy is a favorite choice for most vesical calculi with an obstructed labor [1] [18] [19]. The perception that the bladder mass might be bladder cancer barred immediate cystolithotomy in the index patient.

Though there is no clear consensus on the best management modality of vesical calculus in pregnancy, the handy guidelines on management of urolithiasis in pregnancy are beneficial. Observation withmedical expulsion therapy is usually considered as the first-line therapy for patients with small asymptomatic vesical stone in pregnancy. While second-line therapy such as Transurethral cystolitholapaxy, percutaneous suprapubic cystolitholapaxy, open suprapubic cystolithotomy are preserved for the patients with large bladder stone that presents early in pregnancy or with failed conservative therapy [20]. But patients with large bladder stone presenting with obstructed labor are usually managed with CS and cystolithotomy at the same sitting. Therefore, for a calculus of 536 grams, the single practicable treatment in the center is open suprapubic cystolithotomy through the CS scar. Treatment of the predisposing factor to the calculus formation is fundamental to the prevention of recurrence. Our patient has no intra-operative features suggestive of bladder outlet obstruction; however, chronic cystitis secondary to schistosomiasis was established on histology and she was treated though no clear risk factor for the infestation was established in the patient.

\section{Conclusion}

Prolonged obstructed labor is an uncommon presentation of a giant vesical cal- 
culus. Consequently, the diagnosis needs a high index of suspicion. Clinical and imaging evaluations establish the diagnosis. The calculus extracted weighs $536 \mathrm{~g}$ is the biggest bladder calculus picked as a result of prolonged obstructed labor in the literature.

\section{Conflicts of Interest}

The authors declare no conflicts of interest regarding the publication of this paper.

\section{References}

[1] Sonak, M., Ramteke, S. and Davile, M. (2017) Bladder Stone: A Rare Cause of Obstructed Labour. International Surgery Journal, 4, 2352-2354.

https://doi.org/10.18203/2349-2902.isj20172796

[2] Basler, J. (2017) Bladder Stones. https://emedicine.medscape.com/article/2120102-overview

[3] Farncombe, R. (1935) Foreign Body in the Bladder Associated with Pregnancy. The Lancet, 226, 825-826. https://doi.org/10.1016/S0140-6736(00)47515-X

[4] Cope, E. (1961) Obstructed Labour Due to Vesical Calculus. BJOG: An International Journal of Obstetrics and Gynaecology, 68, 476-478. https://doi.org/10.1111/j.1471-0528.1961.tb02756.x

[5] Ait, B.Y., Aboulfalah, A. and Abbassi, H. (2006) Bladder Stone: Uncommon Cause of Mechanicaldy Stocia. Archives of Gynecology and Obstetrics, 274, 323-324. https://doi.org/10.1007/s00404-006-0163-x

[6] Chakraborty, B., Mondal, P.C., Sahana, R. and Barman, S.C. (2015) A Giant Vesical Stone Causing Impending Rupture of Bladder during Labor. The Journal of Obstetrics and Gynecology of India, 65, 267-270. https://doi.org/10.1007/s13224-014-0543-2

[7] Pricilla, R.A., David, K.V., Venkatesan, S. and Benjamin, S.J. (2013) Early Diagnosis of a Large Vesical Calculus Complicating Pregnancy. Journal of Family Medicine and Primary Care, 2, 88-89.

[8] Cunningham, F.G., Leveno, K.J., Bloom, S.L., Hauth, J.C., Gilstrap, L. and Wenstorm, K.D. (2005) Williams Obstetrics. 22nd Edition, McGraw-Hill, NewYork.

[9] Dave, A., Mathur, P. and Mathuriya, G. (2012) Vesical Calculus: An Uncommon Cause of Obstructed Labor. The Journal of Obstetrics and Gynecology of India, 62, 687-688. https://doi.org/10.1007/s13224-012-0145-9

[10] Rai, L. and Ramesh, K. (1998) Obstructed Labour Due to a Vesicalcal Culus. Obstetrics and Gynecology, 38, 474. https://doi.org/10.1111/j.1479-828X.1998.tb03117.x

[11] Armon, P.J. (1977) Obstructed Labour Due to a Vesical Calculus. British Medical Journal, 2, 498. https://doi.org/10.1136/bmj.2.6085.498

[12] Egwuatu, V.E. (1980) Bladder Calculus with Pregnancy. Journal of Urology, 123, 954-955. https://doi.org/10.1016/S0022-5347(17)56211-4

[13] World Health Organization. Maternal Mortality in 1990-2015. WHO, UNICEF, UNFPA, World Bank Group, and United Nations Population Division Maternal Mortality Estimation Inter-Agency Group. https://www.who.int/gho/maternal_health/countries/nga.pdf

[14] Neer, C.S. (1919) Dystocia from Large Bladder Stone Impacted in the Pelvis. Journal 
of the American Medical Association, 72, 479-480.

https://doi.org/10.1001/jama.1919.02610070017009

[15] Sarma, V. (1960) Vesical Calculus as a Cause of Labour Dystocia, Report of a Case. Postgraduate Medical Journal, 36, 626-628. https://doi.org/10.1136/pgmj.36.420.626

[16] Türk, C., Knoll, T., Petrik, A., Sarica, K., Skolarikos, A., Straub, M., et al. (2016) European Association of Urology 2016 Urolithiasis Guidelines.

[17] Patel, S.J., Reede, D.L., Katz, D.S., Subramaniam, R. and Amorosa, J.K. (2007) Imaging the Pregnant Patient for Nonobstetric Conditions: Algorithms and Radiation Dose Considerations. Radio Graphics, 27, 1705-1722. https://doi.org/10.1148/rg.276075002

[18] Dhingra, S., Baria, J., Nigam, A. and Das, L. (2012) Vesical Calculi: A Rare Cause of Dystocia. Journal of Case Reports, 2, 107-109. https://doi.org/10.17659/01.2012.0033

[19] Seth, S., Malik, S. and Salhan, S. (2002) Vesical Calculus Causing Dystocia. European Journal of Obstetrics and Gynecology and Reproductive Biology, 101, 199-200. https://doi.org/10.1016/S0301-2115(01)00542-5

[20] Fontanilla, B.C. (1946) Surgicalapproach in a Case of Large, Multiple Cystolithiasis Obstructing the Free Passage of Fetal Head. Journal of the Philippine Medical Association, 22, 531-534. 